\title{
Kinematic Analysis and Simulation of an Uncoupled Translational Parallel Mechanism
}

\author{
Yanbin Zhang ${ }^{1,2, *}$ Yifu Zhao ${ }^{1, a} \quad$ Xianling Jing ${ }^{1, b}$ \\ ${ }^{1}$ School of Mechatronics Engineering, Henan University of Science \& Technology, \\ Luoyang, Henan 4710003, PR China, \\ ${ }^{2}$ Center of Machinery Equipment Advanced Manufacturing of Henan Province, \\ Luoyang, Henan 4710003, PR China \\ azyf_zmd@163.com, ${ }^{b}$ jx12048@163.com \\ *yanbin_zh@163.com
}

Keywords: Translational parallel mechanism; Uncoupling; Kinematic analysis; Simulation

\begin{abstract}
A novel uncoupled translational parallel mechanism (UTPM) with three degrees of freedom (3-DOFs) is presented, which is composed of a moving platform connected to a fixed base by three identical kinematic chains. Firstly, kinematic output property of the moving platform is analyzed. Then, both the forward and the inverse analytical solutions of the kinematic problems are discussed in detail. The velocity Jacobian is a $3 \times 3$ diagonal matrix, which implies that there exists a one-to-one corresponding relation between the inputs and the outputs of the parallel mechanism. Finally, the kinematic simulation is carried out by MATLAB and Pro/E software. Simulation results show that the theoretical analysis is correct and feasible.
\end{abstract}

\section{Introduction}

Parallel mechanism (PM), which comprises of a moving platform (MP) connected to a fixed base (FB) by several serial open-single kinematic chains, is the essential part of robot technology and has been extensively studied by many scholars during past decades ${ }^{[1-2]}$. Contrasted with 6-DOFs PM, the limited-DOF PM possesses many advantages in terms of simpler mechanical structure and control system, easier kinematics and dynamic analysis. Due to those superiorities, the limited-DOF $\mathrm{PM}$ owns the expansive application prospect in advanced manufacturing industry and robot industry $^{[3-4]}$.

Translational parallel mechanism (TPM) with 3-DOFs has been concerned specially, which has widely applications in the fields of industrial robots, machine tools, medical robots, and so on. Although the kinematic coupling brings about some merits in terms of higher rigidity and stronger load capacity, it also produces some problems on kinematics analysis and design of control system. Uncoupled parallel mechanism (UPM), however, possesses the special property that one input of the actuator only effect one output of the platform. Therefore, it has been highlights in mechanism field $^{[5-6]}$, recently. Kong and Gosselin ${ }^{[7]}$ proposed a 3-CRR UTPM. Li ${ }^{[8]}$ presented a kind of decoupled TPMs. Zhang ${ }^{[9]}$ synthesized a type of 3-DOFs UTPMs based on reciprocal screw theory.

In this paper, a novel 3-DOFs UTPM is presented, in which the MP is linked to the FB by three serial open-single kinematic chains. Other contents are arranged as follows: firstly, a brief description of architectural design of the PM are performed, Secondly, the kinematic output property of the MP is discussed, thirdly, both the forward and the inverse kinematic problems are analyzed, finally, the simulation of this PM was carried out by MATLAB and Pro/E software, which concludes that the theoretic analysis is correct and verifies that the TPM is uncoupling. 


\section{Design of the parallel mechanism}

Structure of the TPM proposed here is shown in Figure 1. It is composed of three kinematic chains with identical architecture. For the ith chain, it is regarded as $\mathrm{R}_{i 1} / / \mathrm{R}_{i 2} \perp \mathrm{R}_{i 3} / /\left(\perp \mathrm{P}_{i 4} \perp\right) \mathrm{R}_{i 5}$ $(i=1,2,3)$ in sequence from the base to the platform. Here $\mathrm{R}, \mathrm{P}$ denote the revolute and the prismatic joints, respectively, the symbol " //" and " $\perp$ " represent the axes of the neighboring joints being parallel or perpendicular to each other. The assembly conditions of the chains are that the axes of $\mathrm{R}_{i 1}$ joints are normal to each other and intersected together. Similarly, the axes of $\mathrm{R}_{i 5}$ joints must be also satisfied the same condition. Origin $O$ of the global frame Oxyz lies on the intersection point of the axes of $\mathrm{R}_{i 1}$ joints. The axes of $\mathrm{R}_{11}, \mathrm{R}_{21}$ and $\mathrm{R}_{31}$, joints are superimposed with three axes of the global frame, respectively.

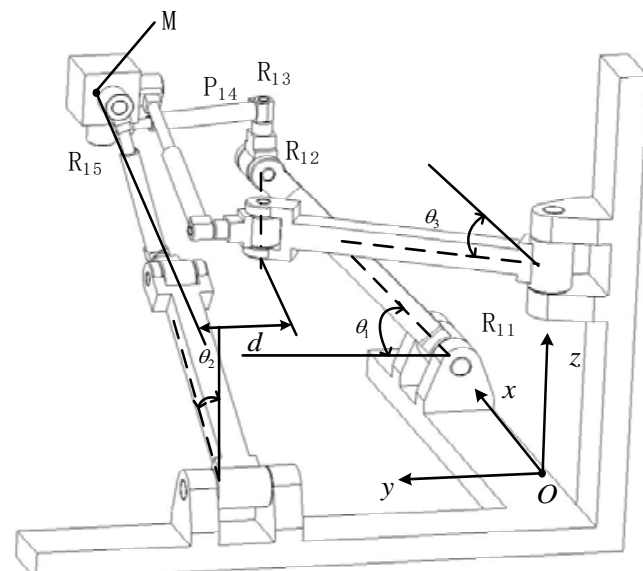

Figure 1 The structure diagram of the UTPM

\section{Mobility analysis}

The output motion of the MP for the PM is determined by the constraints provided by all of kinematic chains. In other words, it is the intersection set of output motion characteristic matrixes of all kinematic chains and can be expressed as

$$
\boldsymbol{M}_{p}=\bigcap_{i=1}^{n} \boldsymbol{M}_{l i}
$$

where $\boldsymbol{M}_{p}$ denotes the output motion characteristic matrix of the MP, $\boldsymbol{M}_{l i}$ is output motion characteristic matrixes associated with the ith kinematic chain, $n$ expresses the mount of the kinematic chains.

Based on Figure 1, the output motion characteristic matrix of the UTPM is obtained, and have

$$
\boldsymbol{M}_{p}=\left[\begin{array}{ccc}
x & y & z \\
\hat{x} & \bullet & \hat{z}
\end{array}\right] \cap\left[\begin{array}{ccc}
x & y & z \\
\hat{x} & \hat{y} & \cdot
\end{array}\right] \cap\left[\begin{array}{ccc}
x & y & z \\
\cdot & \hat{y} & \hat{z}
\end{array}\right]=\left[\begin{array}{ccc}
x & y & z \\
\bullet & \bullet & \cdot
\end{array}\right]
$$

in which the symbol '.' expresses the constant in the motion characteristic matrix. From Eq.(2), we conclude that the MP only possesses three translational DOFs along the axes of the global frame.

\section{Kinematics analysis}

\subsection{Position Analysis}

Position analysis is to determine the relationship between the input displacements of the actuated 
joints and the output displacements of the platform. As for the mechanism shown in Figure 1, to select the centroid $\mathrm{M}$ of the MP as research object, its coordinate is defined as $\mathrm{M}\left(x_{\mathrm{M}}, y_{\mathrm{M}}, z_{\mathrm{M}}\right)$ with respect to the frame $O x y z . d$ is the distance between the axis of $\mathrm{R}_{i 2}$ and the line passing through point $\mathrm{M}$ and parallel to the corresponding axis of the frame. $l$ is the length of the link connected with $\mathrm{R}_{i 1}$ and $\mathrm{R}_{i 2}$ joints.

\subsubsection{Forward position analysis}

The forward position analysis is to compute the position coordinate $\mathrm{M}\left(x_{\mathrm{M}}, y_{\mathrm{M}}, z_{\mathrm{M}}\right)$ of the MP when all generalized input angular displacements $\theta_{i}(i=1,2,3)$ of the actuators are given. According to the structure of mechanism, it is very convenient to find that

$$
\begin{gathered}
x_{\mathrm{M}}=l \sin \theta_{2}+d \\
y_{\mathrm{M}}=l \sin \theta_{3}+d \\
z_{\mathrm{M}}=l \sin \theta_{1}+d
\end{gathered}
$$

\subsubsection{Inverse position analysis}

The inverse position analysis is to find the input angular displacements $\theta_{i}$ of the actuators when the position coordinate $\left(x_{M}, y_{M}, z_{M}\right)$ of the point $\mathrm{M}$ are given. On the basis of Eqs.(3)-(5), yields

$$
\begin{aligned}
& \theta_{1}=\sin ^{-1}\left[\left(z_{\mathrm{M}}-d\right) / l\right] \\
& \theta_{2}=\sin ^{-1}\left[\left(x_{\mathrm{M}}-d\right) / l\right] \\
& \theta_{3}=\sin ^{-1}\left[\left(y_{\mathrm{M}}-d\right) / l\right]
\end{aligned}
$$

\subsection{Velocity Analysis}

Differentiating Eqs.(3)-(5) with respect to time $t$ and rewriting them as matrix form, the direct velocity vector equation can be obtained as follows

$$
\left[\begin{array}{l}
v_{x} \\
v_{y} \\
v_{z}
\end{array}\right]=\left[\begin{array}{ccc}
0 & l \cos \theta_{2} & 0 \\
0 & 0 & l \cos \theta_{3} \\
l \cos \theta_{1} & 0 & 0
\end{array}\right]\left[\begin{array}{l}
\dot{q}_{11} \\
\dot{q}_{21} \\
\dot{q}_{31}
\end{array}\right]
$$

where $\left[v_{x}, v_{y}, v_{z}\right]^{\mathrm{T}}$ and $\left[\dot{q}_{11}, \dot{q}_{21}, \dot{q}_{31}\right]^{\mathrm{T}}$ represent the output velocity vector of the MP and the input angular velocity vector of the actuators, respectively. Clearly, the Jacobian is equivalent to a diagonal matrix. Therefore, there exists a one-to-one corresponding relationship between the inputs and the outputs. That is to say, one output of the platform is only controlled by one input of the actuators and the mechanism shows uncoupled performance throughout the workspace.

\subsection{Acceleration analysis}

According to the method of the second order influence coefficient ${ }^{[10]}$, the acceleration equation can be written as

$$
\boldsymbol{a}_{\mathrm{M}}^{\mathrm{T}}=\dot{\boldsymbol{q}} \boldsymbol{H}_{q}^{\mathrm{M}} \dot{\boldsymbol{q}}^{\mathrm{T}}+\boldsymbol{G}_{q}^{\mathrm{M}} \ddot{\boldsymbol{q}}^{\mathrm{T}}
$$

where $\boldsymbol{a}_{\mathrm{M}}=\left[a_{x}, a_{y}, a_{z}\right]$ denotes the acceleration vector of the PM, $\ddot{\boldsymbol{q}}=\left[\dot{q}_{11}, \dot{q}_{12}, \dot{q}_{13}\right]$ represents the angular acceleration vector of the actuators, $\boldsymbol{G}_{q}^{\mathrm{M}}$ is the first influence coefficient matrix of the velocity equation, i.e., Jacobian matrix, $\boldsymbol{H}_{q}^{\mathrm{M}}$ is the second order influence coefficient matrix, and have

$$
\boldsymbol{H}_{q}^{\mathrm{M}}=\left[\begin{array}{lll}
H_{11} & H_{12} & H_{13} \\
H_{21} & H_{22} & H_{23} \\
H_{31} & H_{32} & H_{33}
\end{array}\right]
$$

in which all of elements in matrix can be calculated, and have, $H_{11}=\left[\begin{array}{llll}-l \sin \theta_{1} & 0 & 0\end{array}\right]^{\mathrm{T}}$, 


$$
\begin{aligned}
& H_{12}=H_{21}=\left[\begin{array}{lll}
0 & 0 & 0
\end{array}\right]^{\mathrm{T}}, \quad H_{22}=\left[\begin{array}{lll}
0 & -l \sin \theta_{2} & 0
\end{array}\right]^{\mathrm{T}}, H_{13}=H_{31}=\left[\begin{array}{lll}
0 & 0 & 0
\end{array}\right]^{\mathrm{T}}, \quad H_{33}=\left[\begin{array}{lll}
0 & 0 & -l \sin \theta_{3}
\end{array}\right]^{\mathrm{T}}, \\
& H_{23}=H_{32}=\left[\begin{array}{lll}
0 & 0 & 0
\end{array}\right]^{\mathrm{T}} .
\end{aligned}
$$

Substituting all known parameters into Eq.(10), acceleration of the point $\mathrm{M}$ will be gotten. After transforming the form of Eq.(10), when the inverse of $\boldsymbol{G}_{q}^{\mathrm{M}}$ exists, the inverse acceleration equation is obtained as well, and

$$
\ddot{\boldsymbol{q}}^{\mathrm{T}}=\boldsymbol{G}_{q}^{\mathrm{M}^{-1}}\left(\boldsymbol{a}_{\mathrm{M}}^{\mathrm{T}}-\dot{\boldsymbol{q}} \boldsymbol{H}_{q}^{\mathrm{M}} \dot{\boldsymbol{q}}^{\mathrm{T}}\right)
$$

\section{Kinematics simulation}

Pro/E software possesses well performance in modeling and simulation of the virtual prototype. Firstly, we use it to establish the 3-D model of the virtual prototype as shown in Fig.1. Then , adding three angular velocity equations to the servo motors, respectively. The kinematic expressions of the actuated joints are as follows, $\dot{q}_{11}=10 \sin (\pi t / 10), \dot{q}_{12}=7.5 \sin (\pi t / 10)$ and $\dot{q}_{13}=12.5 \sin (\pi t / 10)$. At the same time, we adapt that the initial parameters of the actuated joints are selected as $\theta_{1}=\theta_{2}=\theta_{3}=\pi / 4$. Moreover, other structural parameters are also given, such as $d=122.605 \mathrm{~mm}, l=204.680 \mathrm{~mm}$. Simulation results are described in Fig.2, in which (a), (b) and (c) respectively present the position, velocity and acceleration curves.

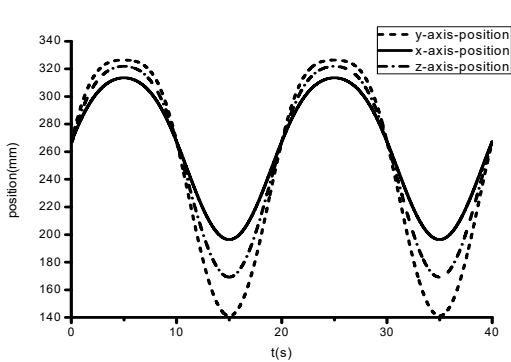

(a)

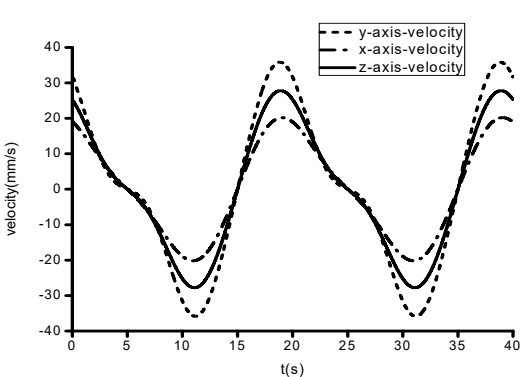

(b)

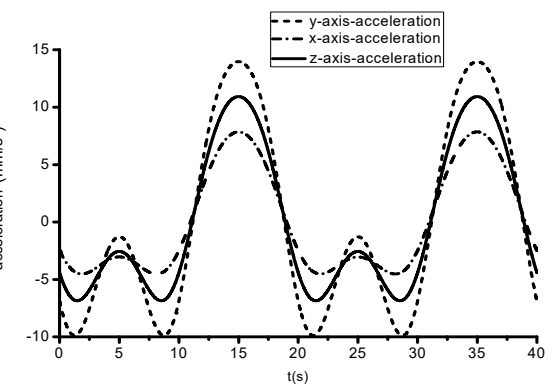

(c)

Figure 2 Kinematic curves carried out by Pro/E

Similarly, all kinematic curves of point $\mathrm{M}$ are also obtained by using MATLAB software, according to the theoretical mathematic models derived above, see Figure 3.

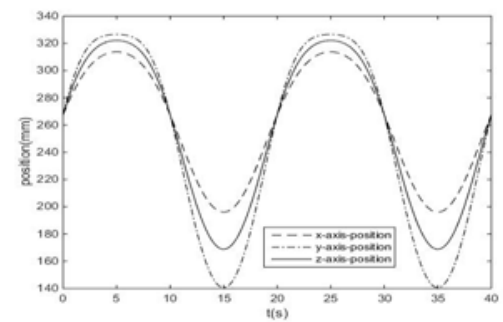

(a)

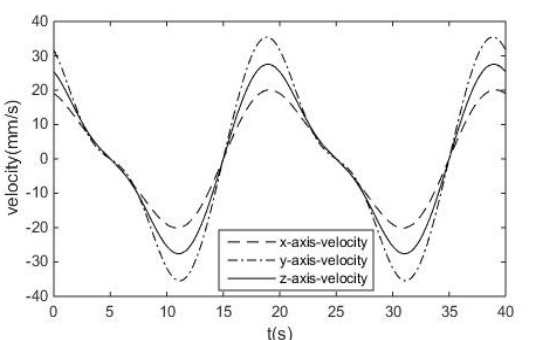

(b)

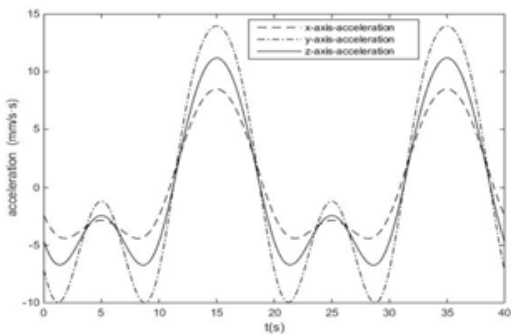

(c)

Figure 3 Kinematic curves carried out by MATLAB

To compare with Figure 3 and Figure 2, it manifests that the kinematic curves carried out by Pro-E and MATLAB software are identical respectively. Therefore, it concludes that the theoretical mathematic models established in this paper are correct.

\section{Conclusion}

This paper presented a novel UTPM, in which three kinematic chains are arranged in the space with orthogonal characteristic. Firstly, the kinematic output property is obtained. Secondly, the 
relationship between input and output motion is achieved, which can achieve that the Jacobian matrix is diagonal. Thirdly, simulation carried out by Pro-E and MATLAB software, it proves the correctness of theoretical analysis, meanwhile, it concludes that the TPM is uncoupling. The UTPM can solve the problem of the kinematics and dynamic analysis and the bigger accumulative error of the MP brought by the kinematic coupling. Simultaneously, it can reduce accumulative error of the MP and simplify the control system. Because of superiority brought by kinematic uncoupling, it possesses a grander prospect in application of automatic production line and intelligent manufacturing system.

\section{Acknowledgments}

This work was supported by the Fundamental Project of Key Scientific Research of Henan Advanced Education (18A460001), Program for Innovative Research Team of Henan University of Science and Technology (2015XTD012), and Program for Postgraduate Innovative Foundation of Henan University of Science and Technology (CXJJ-2016-ZR03).

\section{References}

[1] De la Torre H, Rodriguez-Leal E. Instantaneous Kinematics Analysis via Screw-Theory of a Novel 3-CRC Parallel Mechanism[J]. International Journal of Advanced Robotic Systems, 2016, 13(3): 128.

[2] Rodriguez-Leal E, Dai J S, Pennock G R. A study of the instantaneous kinematics of the 5-RSP parallel mechanism using screw theory[M]. Advances in Reconfigurable Mechanisms and Robots I. Springer London, 2012: 355-369.

[3] De la Torre H, Rodriguez-Leal E. Instantaneous Kinematics Analysis via Screw-Theory of a Novel 3-C RC Parallel Mechanism[J]. International Journal of Advanced Robotic Systems, 2016, 13(3): 128.

[4] Callegari M, Gabrielli A, Ruggiu M. Kineto-elasto-static synthesis of a 3-CRU spherical wrist for miniaturized assembly tasks[J]. Meccanica, 2008, 43(4): 377-389.

[5] Yanbin Zhang, Hongzhao Liu, Xin Wu. Kinematics analysis of a novel parallel manipulator[J]. Mechanism and Machine Theory, 2009, 44(9): 1648-1657.

[6] Chen H, Cao Y, Qing Y L. Type synthesis of fully-decoupled and fully-isotropic 1T1R parallel robotic manipulators[J]. China Mechanical Engineering, 2016, 27(05): 589-595.

[7] Kong X, Gosselin C M. Kinematics and singularity analysis of a novel type of 3-CRR 3-DOF translational parallel manipulator[J]. The International Journal of Robotics Research, 2002, 21(9): 791-798.

[8] Li W, Gao F, Zhang J. R-CUBE, A decoupled parallel manipulator only with revolute joints[J]. Mechanism and Machine Theory, 2005, 40(4): 467-473.

[9] Zhang Y B, Liu H Z, Wu X. Type synthesis of non-singular fully-isotropic translational parallel mechanisms based on theory of reciprocal screw[J]. Chinese Journal of Mechanical Engineering, 2008, 44(10): 83-88.

[10] Huang Z, Zhao Y S, Zhao T S. Advanced Spatial Mechanism[M]. Beijing: Higher Education Press, 2006 